\title{
Screening of potentially crucial genes and regulatory factors involved in epithelial ovarian cancer using microarray analysis
}

\author{
CAN SHI and ZHENYU ZHANG \\ Department of Obstetrics and Gynecology, Beijing Chao-Yang Hospital, \\ Capital Medical University, Beijing 100020, P.R. China
}

Received October 8, 2015; Accepted March 17, 2017

DOI: $10.3892 / \mathrm{ol} .2017 .6183$

\begin{abstract}
The present study aimed to screen potential genes implicated in epithelial ovarian cancer (EOC) and to further understand the molecular pathogenesis of EOC. In order to do this, datasets GSE14407 (containing 12 human ovarian cancer epithelia samples and 12 normal epithelia samples) and GSE29220 (containing 11 salivary transcriptomes from ovarian cancer patients with serous papillary adenocarcinoma and 11 matched controls) were obtained from the Gene Expression Omnibus. Differentially expressed genes (DEGs) within these datasets were screened using the Linear Models for Microarray Data package, and potential gene functions were predicted by functional and pathway enrichment analyses. Additionally, module analysis of protein-protein interaction networks was performed using MCODE software in Cytoscape. The potential microRNAs (miRNAs/miRs) and transcription factors (TFs) regulating DEGs were also analyzed, and the integrated TF-DEG and miRNA-DEG regulatory networks were visualized with Cytoscape. In total, 31 upregulated DEGs and 64 downregulated DEGs were screened. The upregulated DEGs, such as centromere protein $\mathrm{F}(C E N P F)$ and ubiquitin like with PHD and ring finger domains 1 (UHRF1), were significantly associated with the cell cycle and were regulated by the TF nuclear transcription factor Y (NF-Y). CENPF was modulated by miR-373, and UHRF1 was regulated by miR-146a. The downregulated DEGs, such as aldehyde dehydrogenase 1 family member A2 (ALDH1A2), were distinctly involved in the response to estrogen stimulus and modulated by tumor protein 53 (TP53); protocadherin 9 ( $P C D H 9)$ was regulated by TP53, miR-92b-3p and miR-137. The DEGs, including $C E N P F, U H R F 1, A L D H 1 A 2$ and $P C D H 9$, and a set of gene regulators, including all $N F Y$ genes, $T P 53$, miR-373, miR-146a,
\end{abstract}

Correspondence to: Dr Zhenyu Zhang, Department of Obstetrics and Gynecology, Beijing Chao-Yang Hospital, Capital Medical University, 8 Gongti South Road, Chaoyang, Beijing 100020, P.R. China

E-mail: zhenyuzhang64@163.com

Key words: epithelial ovarian cancer, differentially expressed gene, regulatory network, transcription factor, microRNA
miR-92b-3p and miR-137, may be involved in the pathogenesis of EOC.

\section{Introduction}

Epithelial ovarian cancer (EOC), the primary gynecological cause of oncological mortality, accounts for $4 \%$ of all cancer types in women (1). Despite medical and surgical advances, patients with advanced EOC continue to endure poor long-term survival rates (2). This is largely a result of the limited understanding of the molecular mechanisms of EOC pathogenesis.

Recently, marked achievements in the investigation of molecular mechanisms of EOC have been made. In ovarian cancer cells, the Janus kinase 2/signal transducer and activator of transcription 3 pathway was found to be constitutively active and directly dependent on the activation of epidermal growth factor receptor (EGFR) or interleukin 6 receptor (IL-6R) (3); this pathway is required to sustain EGF-induced epithelial-mesenchymal transition-associated phenotypes in ovarian cancer cells (4). A previous study also demonstrated that the Kirsten rat sarcoma 2 viral homolog (KRAS)-V-Raf murine sarcoma viral oncogene homolog B1 (BRAF)-mitogen-activated protein kinase kinase 1 (MEK)-mitogen-activated protein kinase 1 (MAPK) pathway has a key biological role in the development of serous EOC tumors, and activating mutations in KRAS or BRAF result in the constitutive activation of MAPK-mediated signaling (5). Mutations in BRCA1/2 are frequently identified in high-grade serous ovarian cancer; these mutations sensitize EOC patients to the inhibition of poly (ADP-ribose) polymerase-1, increasing the number of patients who benefit (6). Furthermore, low expression of the microRNA (miRNA/miR) miR-100 is associated with the shorter overall survival times of EOC patients; miR-100 affects the growth of EOC cells by post-transcriptionally regulating polo-like kinase 1 expression (7). A previous study demonstrated that overexpression of miR-193a and miR-193b activates caspase 3/7, leading to apoptotic cell death in EOC A2780 cells (8).

Ovarian surface epithelia cells have long been hypothesized to be crucial progenitors of serous EOC (9). In 2009, Bowen et al (10) revealed that differentially expressed genes (DEGs) of human ovarian surface epithelial cells are implicated in the cell-cycle pathway, as well as the WNT, hedgehog and retinoid pathways, which had previously been implicated in the development of EOC. In 2012, Lee et al (11) observed 
that the presence of a combination of five genes (1-acylglycerol-3-phosphate $\mathrm{O}$-acyltransferase $1, \beta$-2-microglobulin, immediate early response 3 , interleukin $1 \beta$ and brain abundant membrane attached signal protein 1) in the saliva had the robust ability to detect ovarian cancer, based on the highest area under the curve value from a receiver operating characteristic plot. The study by Lee et al (11) demonstrated that RNA signatures in saliva acted as biomarkers for the detection of ovarian cancer with high specificity and sensitivity; however, the study only used a single sample source in its analysis and did not investigate regulatory mechanisms involving transcription factors (TFs) or miRNAs. Therefore, more potential molecular mechanisms of EOC pathogenesis must be revealed.

In the present study, two gene expression profile datasets, GSE14407 deposited by Bowen et al (10) and GSE29220 deposited by Lee et al (11), were combined to identify potential key genes and their regulators associated with the pathogenesis of EOC. DEGs between EOC and control samples were screened for, and their functions were analyzed using Gene Ontology (GO) functional analysis and pathway enrichment analysis. A protein-protein interaction (PPI) network was the constructed for these DEGs. Functional enrichment analysis of genes in the PPI network modules was also performed, and potential regulatory TFs and miRNAs of these DEGs were predicted. This microarray analysis may be conducive to providing novel information for the study of EOC pathogenesis and could provide potential biomarkers for the therapy of EOC.

\section{Materials and methods}

Affymetrix microarray data. The gene expression profile data of GSE14407 (10) and GSE29220 (11) were obtained from the Gene Expression Omnibus (GEO, http://www.ncbi.nlm .nih.gov/geo/) database, which was based on the platform of the GPL570 [HG-U133_Plus_2] Affymetrix Human Genome U133 Plus 2.0 Array (Affymetrix, Inc., Santa Clara, CA, USA). The GSE14407 dataset contains 12 samples of epithelial cells from patients with serous papillary ovarian adenocarcinomas and 12 normal human ovarian surface epithelial cell samples. GSE29220 contains 11 salivary transcriptomes from ovarian cancer patients with serous papillary adenocarcinoma and 11 matched controls.

CEL files and probe annotation files were downloaded and the two datasets were combined into one matrix expression profile. The batch deviation (12) in the gene expression data of all samples was wiped out by ComBat order in the surrogate variable analysis package in $\mathrm{R}$ (version 3.22.0; http://www .bioconductor.org/packages/release/bioc/html/sva.html) (13). The data were then preprocessed using background correction, quantile normalization and expression calculation using the preprocessCore package in $\mathrm{R}$ (version 1.36.0; http://www .bioconductor.org/packages/release/bioc/html/preprocess-

Core.html) (14). Afterwards, probe IDs were translated into gene symbols. If one gene symbol was matched by multiple probe IDs, the mean expression value was selected as the expression level of the gene.

$D E G$ screening. Genes that differed significantly in their expression in EOC samples were identified by the Linear Models for Microarray Data package (version 3.30.13; http://www.bioconductor.org/packages/release/bioc/html/ limma.html) (15). The raw P-value was adjusted using the Benjamini-Hochberg method (16) and only the genes with a $\log _{2}$ Fold changel $>1$ and an adjusted P-value $<0.05$ were identified as DEGs in ovarian cancer samples.

GO functional and pathway enrichment analyses. The screened DEGs were submitted to the Database for Annotation, Visualization and Integrated Discovery (DAVID) (17) for GO functional analysis and Kyoto Encyclopedia of Genes and Genomes (18) pathway enrichment analysis, with a cut-off of $\mathrm{P}<0.05$.

PPI network construction. The Search Tool for the Retrieval of Interacting Genes (http://string-db.org/) (19) was used to analyze the PPIs for DEGs by calculating their combined score; a score $>0.4$ was chosen as the cut-off point. PPI networks of upregulated and downregulated DEGs were then visualized by Cytoscape (version 3.4.0; http://cytoscape.org/) (20). Hub proteins (the essential proteins in PPI networks, which have higher degrees) (21) were screened by counting the degree of connectivity of each node in the network. In the network, a node represents a protein (gene) and lines represent the interactions of the proteins. The 'degree' of each node refers to the number of nodes that interact with this node. The larger the degree is, the closer the connections with other nodes are.

Screening and analysis of relevant network modules. On the basis of MCODE analysis (22) of original PPI networks, the network modules were obtained with a cut-off criterion of an MCODE score of $>5$. In order to achieve a better understanding of the function of genes in modules at the molecular level, functional annotation was performed using DAVID and the functional enrichment network was visualized using the plug-in enrichment map in Cytoscape (version 3.4.0; http:// cytoscape.org/) (23).

Construction of integrated TF-DEG regulatory network and miRNA-DEG regulatory network. The University of California Santa Cruz database (http://genome.ucsc.edu/) (24) was used to obtain information on the associations between DEGs and related TFs. The integrated regulatory networks containing TFs with the 5 highest degrees and their corresponding upregulated or downregulated DEGs were then respectively visualized by Cytoscape (version 3.4.0, http://cytoscape.org/).

The common miRNAs predicted to be expressed by the databases of miRecords (http://c1.accurascience.com/miRecords/) (25), TarBase (http://diana.imis.athena-innovation.gr/ DianaTools/index.php?r=tarbase/index) (26) and TargetScan (http://www.targetscan.org/) (27) were selected for the DEGs. Subsequently, the upregulated and downregulated miRNA-DEG regulatory networks were respectively visualized using Cytoscape (version 3.4.0, http://cytoscape.org/).

\section{Results}

Identification of DEGs. After data preprocessing, 20,927 probes were obtained. On the basis of the cut-off criteria, 95 DEGs, including 31 upregulated DEGs and 64 downregulated DEGs, were screened. 


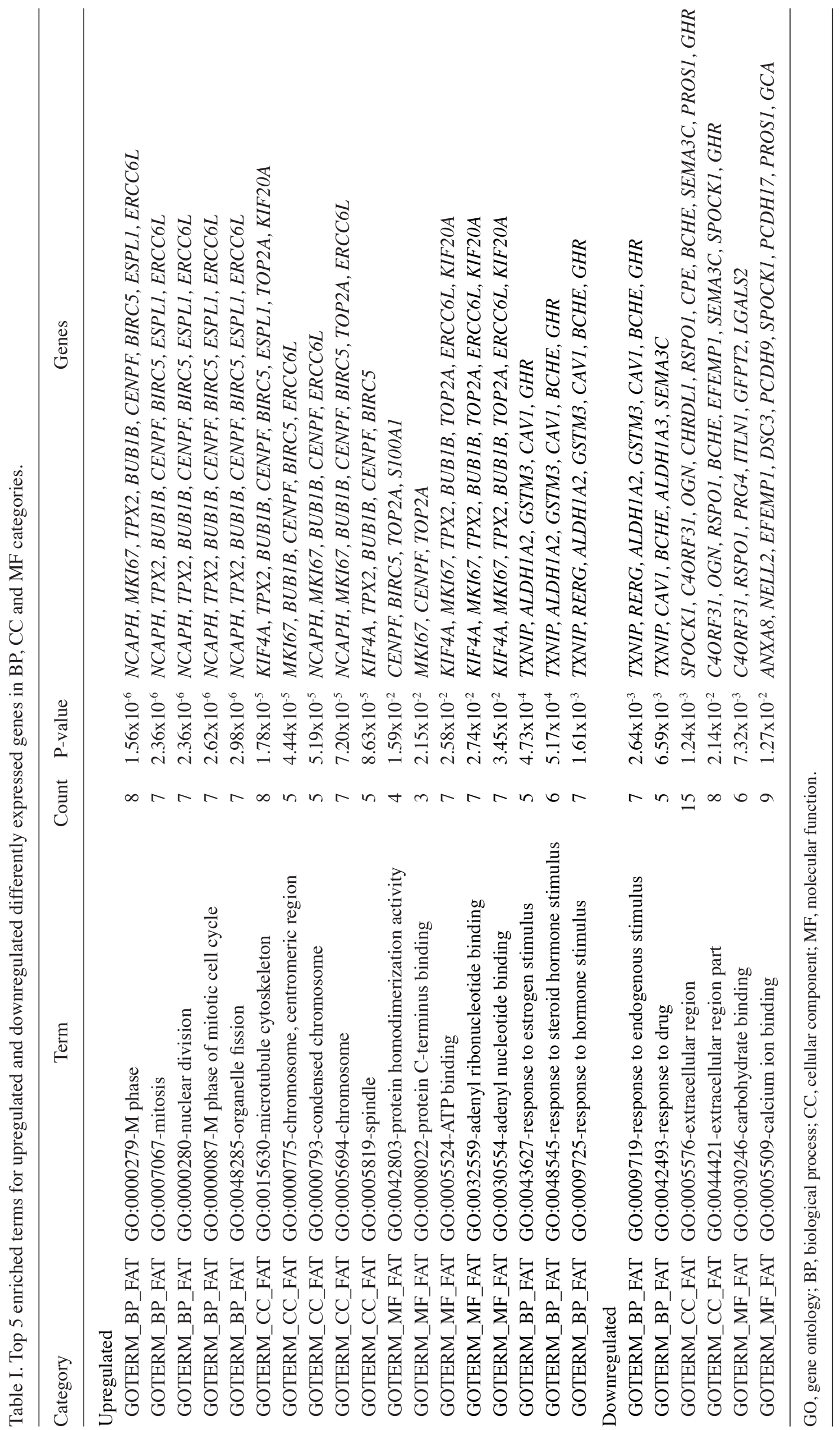


Table II. Pathway enrichment analysis of upregulated and downregulated differently expressed genes.

\begin{tabular}{llcll}
\hline Category & \multicolumn{1}{c}{ Term } & Count & P-value & \multicolumn{1}{c}{ Genes } \\
\hline Upregulated & hsa04310: Wnt signaling pathway & 3 & $1.67 \times 10^{-2}$ & SOX17, FZD2, WNT7A \\
Downregulated & hsa00982: Drug metabolism & 4 & $3.51 \times 10^{-4}$ & GSTM3, ALDH1A3, AOX1, ADH1C \\
& hsa00350: Tyrosine metabolism & 3 & $4.57 \times 10^{-3}$ & ALDH1A3, AOX1, ADH1C \\
& hsa00980: Metabolism of & 3 & $8.36 \times 10^{-3}$ & GSTM3, ALDH1A3, ADHIC \\
& xenobiotics by cytochrome P450 & & & \\
\end{tabular}
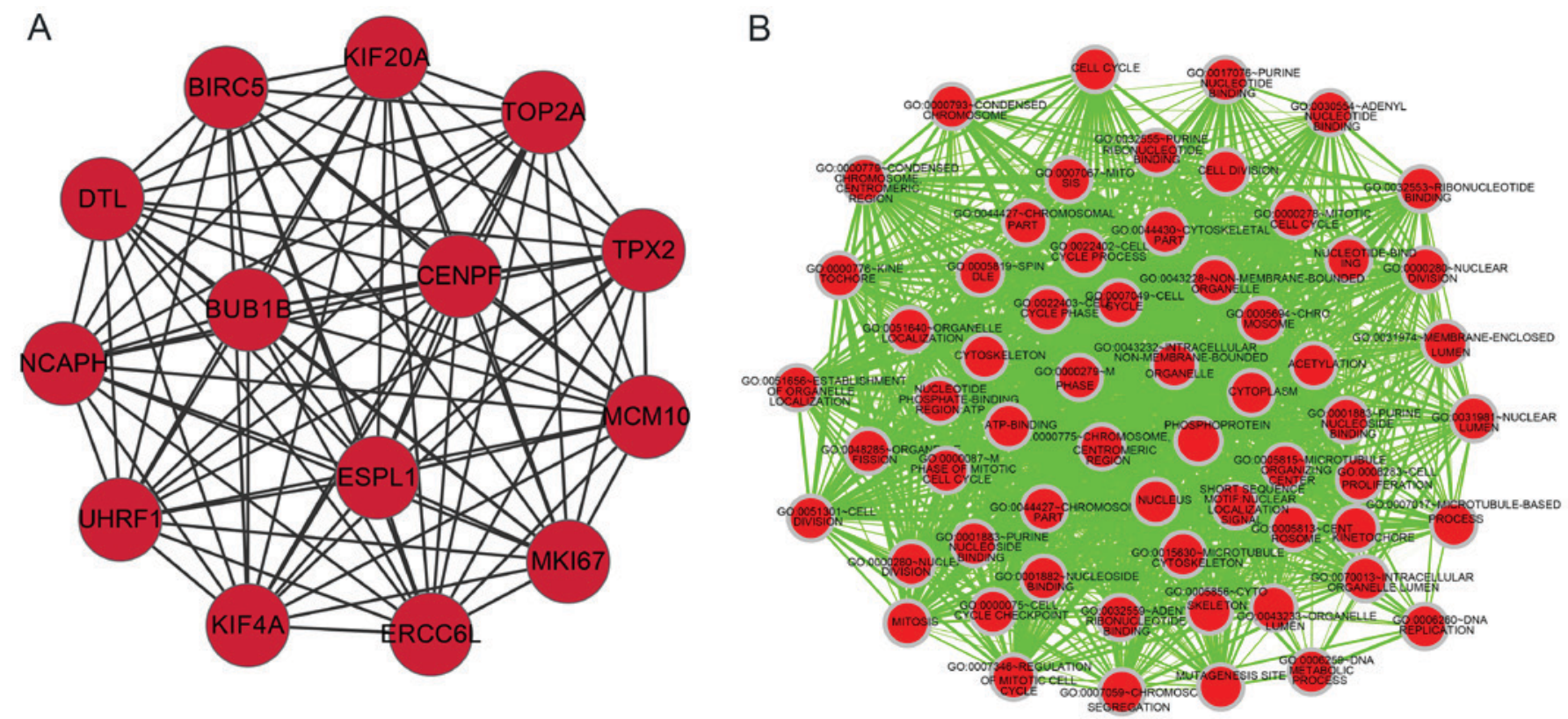

Figure 1. Networks for upregulated genes. (A) The network module for upregulated genes. Each node represents a gene. (B) Functional enrichment network for the upregulated module. Each node represents a functional term for upregulated genes; the width of each line represents the degree of overlap of the gene sets of the two terms at the ends.

Enrichment analysis of upregulated and downregulated DEGs. To reveal the functions of DEGs, GO functional and pathway enrichment analyses were conducted. Upregulated DEGs, including non-SMC condensin I complex subunit $\mathrm{H}$, BUB1 mitotic checkpoint serine/threonine kinase B, CENPF and baculoviral IAP repeat containing 5 (BIRC5), were significantly enriched in the following functional terms: $M$ phase $\left(\mathrm{P}=1.56 \times 10^{-6}\right)$, mitosis $\left(\mathrm{P}=2.36 \times 10^{-6}\right)$, microtubule cytoskeleton $\left(\mathrm{P}=1.78 \times 10^{-5}\right)$, chromosome $\left(\mathrm{P}=4.44 \times 10^{-5}\right)$ and ATP binding $\left(\mathrm{P}=2.58 \times 10^{-2}\right)$ (Table I). Meanwhile, several DEGs in the Wnt signaling pathway (SRY-box 17, frizzled class receptor 2 and Wnt family member 7a) were clearly enriched $\left(\mathrm{P}=1.6678 \times 10^{-2}\right)$ (Table II).

Downregulated DEGs, such as ALDH1A2 and growth hormone receptor $(G H R)$, were distinctly involved in the estrogen stimulus response $\left(\mathrm{P}=4.73 \times 10^{-4}\right)$; these DEGs, including SPARC/osteonectin, cwcv and kazal like domains proteoglycan 1 (SPOCK1) and GHR, were primarily associated with the extracellular region $\left(\mathrm{P}=1.241 \times 10^{-3}\right)$, and DEGs (e.g., $P C D H 9$ and $S P O C K 1$ ) were associated with calcium ion binding $\left(\mathrm{P}=1.2652 \times 10^{-2}\right)$ (Table I). $A L D H 1 A 3$ and $A D H 1 C$ were markedly enriched in three pathways, including drug metabolism $\left(\mathrm{P}=3.51 \times 10^{-4}\right)$, tyrosine metabolism $\left(\mathrm{P}=4.571 \times 10^{-3}\right)$ and metabolism of xenobiotics by cytochrome $\mathrm{P} 450\left(\mathrm{P}=8.375 \times 10^{-3}\right)$ (Table II).

Analysis of network module in PPI network. To investigate the interactions of DEGs further, PPIs of upregulated and downregulated DEGs were respectively analyzed and the PPI network modules were then screened. On the basis of the analysis of PPI networks for upregulated and downregulated DEGs, only one significant network module for upregulated DEGs was screened. The DEGs UHRF1 and CENPF interacted with each other (Fig. 1A). To investigate the functions of genes in the network module, functional enrichment analysis for the upregulated module was performed. The genes in the module were primarily enriched in cell proliferation functions. A set of DEGs, including UHRF1 and CENPF, mainly participated in the cell cycle, mitosis and ATP binding (Fig. 1B).

Analysis of the integrated TF-DEG regulatory networks. To investigate regulators that modulated the DEGs in EOC further, the TFs that regulated upregulated and downregulated DEGs were analyzed, and TF-DEG regulatory networks were constructed. In the upregulated regulatory network, expression of CENPF was regulated by the TFs NFY; UHRF1 was 

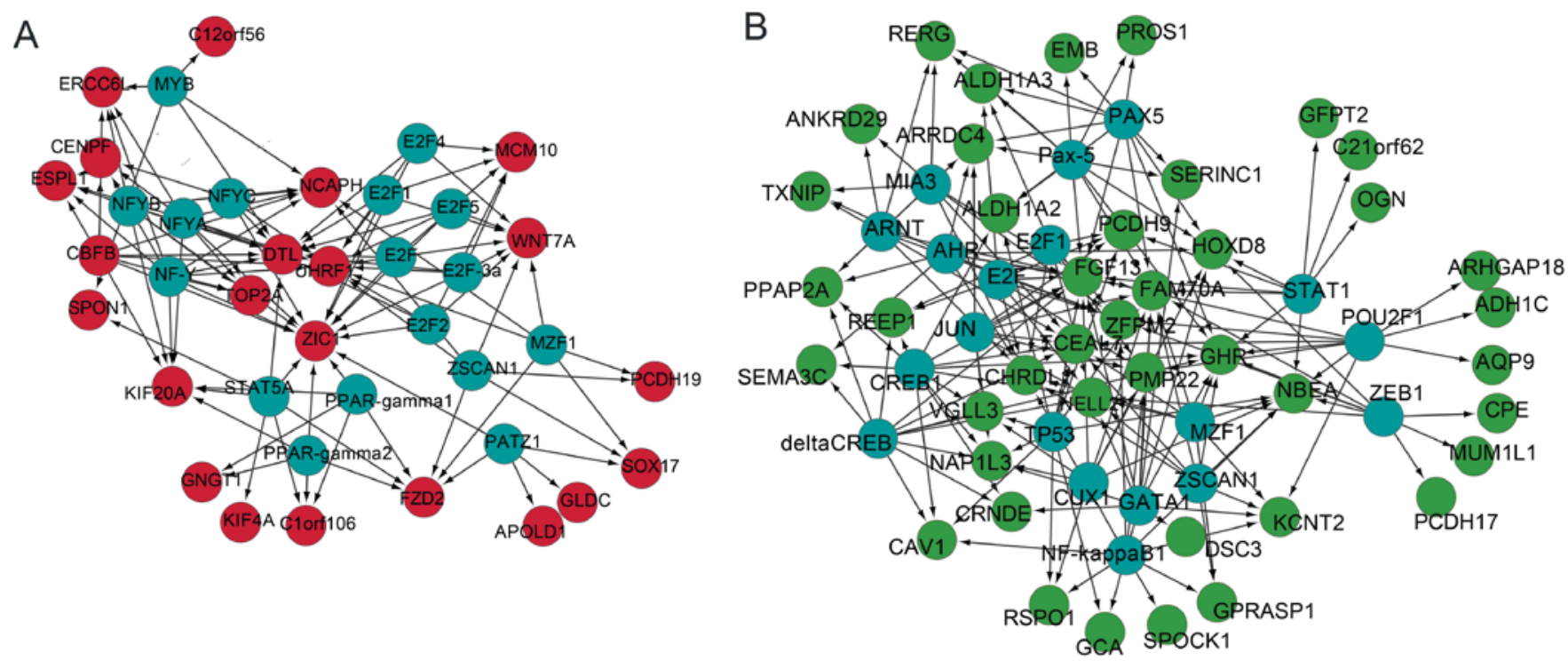

Figure 2. Integrated TF-DEG regulatory networks for upregulated and downregulated genes. (A) The TF-DEG regulatory network for upregulated genes. (B) The integrated TF-DEG regulatory network for downregulated genes. Red nodes represent upregulated genes; green nodes represent downregulated genes; blue nodes represent TFs with the 5 highest degrees. TF, transcription factor; DEG, differentially expressed gene.

modulated by all $E 2 F$ genes and $N F Y$; Zic family member 1 (ZICl) was targeted by E2F and $N F Y$ (Fig. 2A). In the downregulated regulatory network, $A L D H 1 A 2$ and $P C D H 9$ were regulated by TP53. PCDH9 was also regulated by $E 2 F, E 2 F 1$ and $N F Y$ (Fig. 2B).

Analysis of the integrated miRNA-DEG regulatory network. To investigate the associations between DEGs and miRNAs further, miRNA-DEG regulatory networks were constructed. In the upregulated regulatory network, the gene ZICl was regulated by seven miRNAs, including miR-543, miR-23c, miR-23a-3p and miR-514a-3p. Gene UHRFI was regulated by miR-146a and miR-124. Expression of the DEGs BIRC5, $C E N P F$, denticleless E3 ubiquitin protein ligase homolog and minichromosome maintenance 10 replication initiation factor were modulated by miR-203, miR-373, miR-215 and miR-146a, respectively (Fig. 3A).

In the downregulated regulatory network, DEGs, such as zinc finger protein, FOG family member 2 and neurobeachin, were primarily regulated by miRNAs, including miR-300 and miR-153; $P C D H 17$ was primarily modulated by miRNAs, including miR-30a-5p, miR-30b-5p and miR-30d-5p; and $P C D H 9$ was regulated by multiple miRNAs, including miR-32-5p, miR-137 and miR-92a-3p (Fig. 3B).

\section{Discussion}

EOC is the leading cause of mortality in gynecological malignancies (1). In the present study, using the combined analysis of two microarray datasets, 31 genes were identified as being markedly upregulated and 64 downregulated in EOC samples compared with healthy controls.

According to the functional analysis of upregulated module genes, a series of DEGs, including CENPF and UHRF1, were primarily enriched in the cell cycle. CENPF encodes a protein that associates with the centromere-kinetochore complex, which is part of the nuclear matrix during the G2 phase of interphase (28). The kinetochore is a large complex of proteins and associated centromeric DNA that is essential in mitosis (29). CENPF encodes centromere protein $\mathrm{F}$, which drives ovarian cancer growth through regulation of the cell cycle (30). It has been reported that $C E N P F$ is differentially expressed in EOC cells upon the overexpression or knockdown of downstream of tyrosine kinase 1 (31). Furthermore, a recent study has reported that overexpression of CENPK, a homolog of $C E N P F$, is associated with poorer patient survival (32). UHRF1 encodes a member of a subfamily of RING-finger type E3 ubiquitin ligases, which can promote G1/S transition by binding to specific DNA sequences and recruiting a histone deacetylase to regulate gene expression (33). UHRF1 is required for tumor cell proliferation and acts as a dominant effector of cell growth (34). A recent study showed that expression of UHRF1 is higher in ovarian cancer tissue than that in adjacent healthy tissues (35), which is consistent with the results of the present study. This suggests that the genes associated with the cell cycle, such as $C E N P F$ and $U H R F 1$, may play key roles in the process of EOC. Furthermore, $C E N P F$ and $U H R F 1$ were predicted to be regulated by the transcription factor NF-Y. NFYA, NFYB and $N F Y C$ encode NF-Y, a heterotrimeric protein composed of three subunits, NF-YA, NF-YB and NF-YC (36). The NF-Y complex supports the basal transcription of regulatory genes that are responsible for cell-cycle progression, among which are mitotic cyclin complexes (37). A previous study showed that NF-Y regulates mitosis-associated genes, such as CENPF, in multiple cancer types (38). NF-Y is a pivotal regulator of enhancer of zeste 2 polycomb repressive complex 2 subunit expression and is essential for EOC cell proliferation (39). In addition, $C E N P F$ was found to be regulated by miR-373 in the present study. It has been reported that miR-373 expression is downregulated in human EOC and that silencing of miR-373 expression leads to the increased migration and invasion of 
A
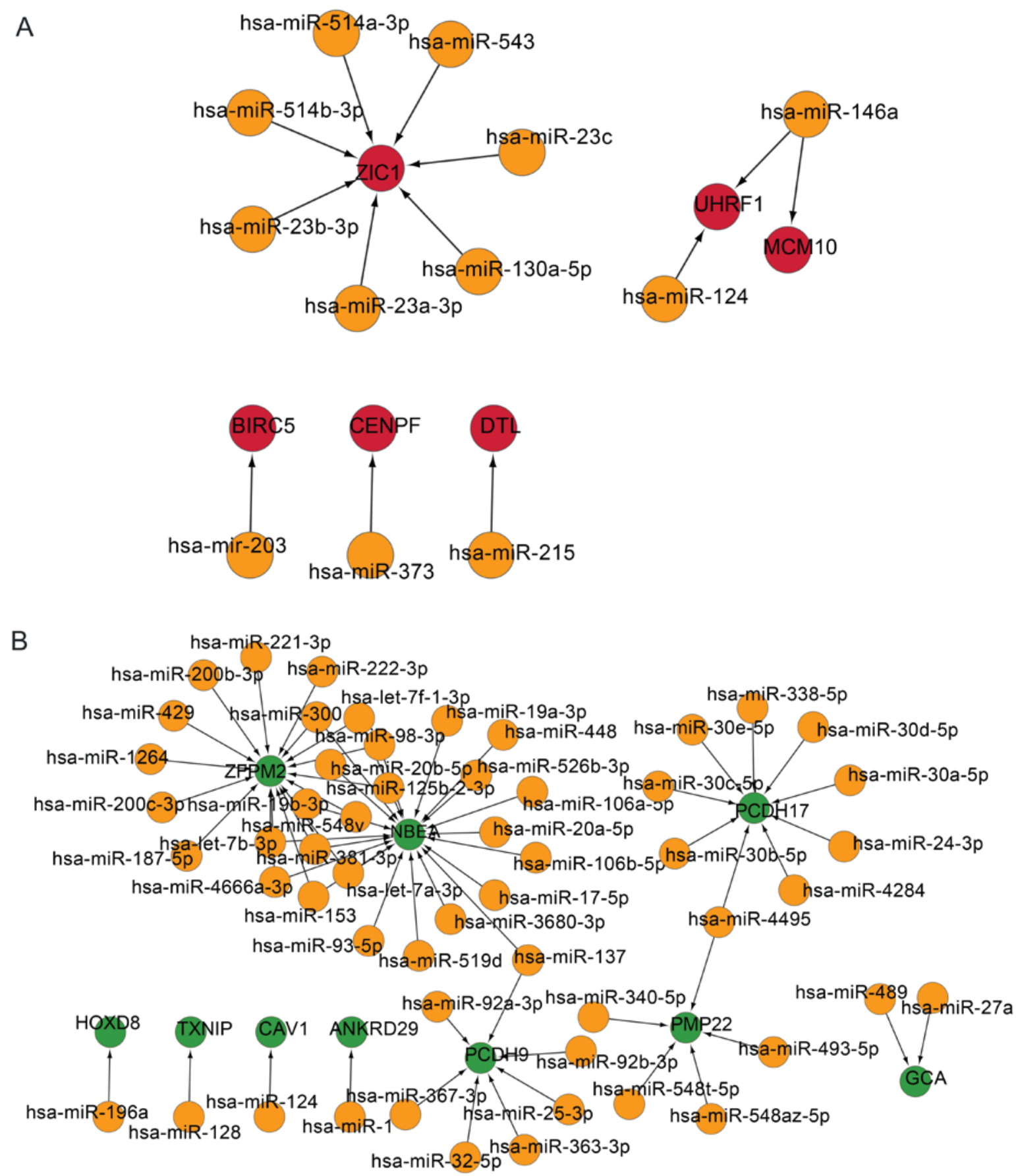

Figure 3. Integrated miRNA-DEG regulatory network for upregulated and downregulated genes. (A) The miRNA-DEG regulatory network for upregulated genes. (B) The integrated miRNA-DEG regulatory network for downregulated genes. Red nodes represent upregulated genes; green nodes represent downregulated genes; orange nodes represent miRNAs associated with genes. miRNA, microRNA; DEG, differentially expressed gene.

EOC cells (40). In the present study, UHRF1 was modulated by miR-146a. Another study showed that ovarian cancer patients with the $\mathrm{C}$ variant allele of miR-146a may have high levels of mature miR-146a (41). Changes in miR-146a expression and/or binding have also been implicated in the metastatic and proliferative response associated with the development of ovarian cancer (41). Collectively, CENPF and UHRF1 may play pivotal roles in the cell cycle and migration in EOC, via the regulation of expression of $N F Y$ and miRNAs, including miR-373 and miR-146a.

With regard to the downregulated DEGs, ALDH1A2 and PCDH9 were regulated by the TF TP53. Genetic alterations to TP53 serve a vital role in ovarian cancer development and progression, as they promote ovarian cancer epithelial cell survival and proliferation (42). However, wild-type TP53 is expressed in ovarian serous carcinomas, particularly in patients with high-grade serous ovarian carcinomas (43), who experience significantly shorter survival times and higher chemoresistance than those with mutated TP53 (44). ALDH1A2 encodes a member of the aldehyde dehydrogenase 1 family, which converts retinaldehyde to retinoic acid, a known marker of lineage-specific stem cells (45). In the present study, $A L D H 1 A 2$ was significantly associated with the estrogen stimulus response. Estrogen has been implicated 
in the etiology and progression of serous ovarian carcinoma by inducing the expression of genes targeted by canonical estrogen receptor $\alpha$ (46), which has been revealed to serve an important role in ovarian cancer development; its expression is a marker of better prognosis (47). $\mathrm{PCDH} 9$ encodes a member of the protocadherin family (and cadherin superfamily) of transmembrane proteins that contain cadherin domains (48). Cadherins can modulate cell adhesion by trans-homodimerization between their membrane-distal EC1 domains that extend from apposed cells and gather at intercellular adherens junctions (49). Cell adhesion plays a notable role in cancer progression and metastasis (50). The intercellular interactions between cancer cells and the endothelium determine the metastatic spread of the disease (50). $\mathrm{PCDH} 9$ expression has been observed in ovarian cancer cells (51). In the present study, $\mathrm{PCDH} 9$ was also found to be regulated by miR-92b-3p and miR-137. miR-92b-3p and miR-137 have been reported to exhibit altered expression levels in ovarian tumor cells $(52,53)$. Taken together, $A L D H 1 A 2$ and $P C D H 9$ may be important in the progression of EOC through the regulation of TP53 or miR-92b-3p and miR-137.

In conclusion, a set of 31 upregulated and 64 downregulated genes (compared with healthy controls) were identified in EOC samples. Among them, upregulated genes that are associated with the cell cycle, including CENPF and UHRF1, and downregulated genes, including $A L D H 1 A 2$ and $P C D H 9$, may be implicated in the progress of EOC via the regulation of TFs, such as NFY and TP53, and miRNAs, such as miR-373, miR-146a, miR-92b-3p and miR-137. The findings of the present study may contribute to a greater understanding of the pathogenesis of EOC; however, these results require future experimental confirmation.

\section{References}

1. Jemal A, Siegel R, Xu J and Ward E: Cancer statistics, 2010. CA Cancer J Clin 60: 277-300, 2010.

2. Marchetti C, Pisano C, Facchini G, Bruni GS, Magazzino FP, Losito S and Pignata S: First-line treatment of advanced ovarian cancer: Current research and perspectives. Expert Rev Anticancer Ther 10: 47-60, 2010.

3. Sansone $\mathrm{P}$ and Bromberg J: Targeting the interleukin-6/Jak/stat pathway in human malignancies. J Clin Oncol 30: 1005-1014, 2012.

4. Colomiere M, Ward AC, Riley C, Trenerry MK, CameronSmith D, Findlay J, Ackland L and Ahmed N: Cross talk of signals between EGFR and IL-6R through JAK2/STAT3 mediate epithelial-mesenchymal transition in ovarian carcinomas. Br J Cancer 100: 134-144, 2009.

5. Mayr D, Hirschmann A, Löhrs U and Diebold J: KRAS and BRAF mutations in ovarian tumors: A comprehensive study of invasive carcinomas, borderline tumors and extraovarian implants. Gynecol Oncol 103: 883-887, 2006.

6. Hennessy B, Timms KM, Carey MS, Gutin A, Meyer LA, Flake DD II, Abkevich V, Potter J, Pruss D, Glenn P, et al: Somatic mutations in BRCA1 and BRCA2 could expand the number of patients that benefit from poly (ADP ribose) polymerase inhibitors in ovarian cancer. J Clin Oncol 28: 3570-3576, 2010.

7. Peng DX, Luo M, Qiu LW, He YL and Wang XF: Prognostic implications of microRNA-100 and its functional roles in human epithelial ovarian cancer. Oncol Rep 27: 1238-1244, 2012.

8. Nakano H, Yamada Y, Miyazawa T and Yoshida T: Gain-of-function microRNA screens identify miR-193a regulating proliferation and apoptosis in epithelial ovarian cancer cells. Int J Oncol 42: 1875-1882, 2013.

9. Auersperg N, Wong AS, Choi KC, Kang SK and Leung PC: Ovarian surface epithelium: Biology, endocrinology, and pathology. Endocr Rev 22: 255-288, 2001.
10. Bowen NJ, Walker LD, Matyunina LV, Logani S, Totten KA, Benigno BB and McDonald JF: Gene expression profiling supports the hypothesis that human ovarian surface epithelia are multipotent and capable of serving as ovarian cancer initiating cells. BMC Med Genomics 2: 71, 2009.

11. Lee YH, Kim JH, Zhou H, Kim BW and Wong DT: Salivary transcriptomic biomarkers for detection of ovarian cancer: For serous papillary adenocarcinoma. J Mol Med (Berl) 90: 427-434, 2012.

12. Johnson WE, Li C and Rabinovic A: Adjusting batch effects in microarray expression data using empirical Bayes methods. Biostatistics 8: 118-127, 2007.

13. Leek JT and Storey JD: A general framework for multiple testing dependence. Proc Natl Acad Sci USA 105: 18718-18723, 2008.

14. Bolstad BM, Irizarry RA, Astrand M and Speed TP: A comparison of normalization methods for high density oligonucleotide array data based on variance and bias. Bioinformatics 19: 185-193, 2003.

15. Smyth GK: Linear models and empirical bayes methods for assessing differential expression in microarray experiments. Stat Appl Genet Mol Biol 3: Article3, 2004.

16. Benjamini Y and Hochberg Y: Controlling the false discovery rate: A practical and powerful approach to multiple testing. J Royal Statist Society Series B Methodol 57: 289-300, 1995.

17. Huang DW, Sherman BT, Tan Q, Collins JR, Alvord WG, Roayaei J, Stephens R, Baseler MW, Lane HC and Lempicki RA: The DAVID gene functional classification tool: A novel biological module-centric algorithm to functionally analyze large gene lists. Genome Biol 8: R183, 2007.

18. Kanehisa M and Goto S: KEGG: Kyoto encyclopedia of genes and genomes. Nucleic Acids Res 28: 27-30, 2000.

19. von Mering C, Huynen M, Jaeggi D, Schmidt S, Bork P and Snel B: STRING: A database of predicted functional associations between proteins. Nucleic Acids Res 31: 258-261, 2003.

20. Kohl M, Wiese S and Warscheid B: Cytoscape: Software for visualization and analysis of biological networks. In: Data Mining in Proteomics, Springer, pp291-303, 2011.

21. He $X$ and Zhang $\mathrm{J}$ : Why do hubs tend to be essential in protein networks? PLoS Genet 2: e88, 2006.

22. Bader GD and Hogue CW: An automated method for finding molecular complexes in large protein interaction networks. BMC Bioinformatics 4: 2, 2003.

23. Merico D, Isserlin R, Stueker O, Emili A and Bader GD: Enrichment map: A network-based method for gene-set enrichment visualization and interpretation. PLoS One 5: e13984, 2010.

24. Karolchik D, Hinrichs AS, Furey TS, Roskin KM, Sugnet CW, Haussler D and Kent WJ: The UCSC table browser data retrieval tool. Nucleic Acids Res 32: D493-D496, 2004.

25. Papadopoulos GL, Reczko M, Simossis VA, Sethupathy P and Hatzigeorgiou AG: The database of experimentally supported targets: A functional update of TarBase. Nucleic Acids Res 37: D155-D158, 2009.

26. Garcia DM, Baek D, Shin C, Bell GW, Grimson A and Bartel DP. Weak seed-pairing stability and high target-site abundance decrease the proficiency of 1sy- 6 and other microRNAs. Nat Struct Mol Biol 18: 1139-1146, 2011.

27. Xiao F, Zuo Z, Cai G, Kang S, Gao X and Li T: miRecords: An integrated resource for microRNA-target interactions. Nucleic Acids Res 37: D105-D110, 2009.

28. Sayer JA, Otto EA, O'Toole JF, Nurnberg G, Kennedy MA, Becker C, Hennies HC, Helou J, Attanasio M, Fausett BV, et al: The centrosomal protein nephrocystin-6 is mutated in Joubert syndrome and activates transcription factor ATF4. Nat Genet 38: 674-681, 2006

29. Yuen KW, Montpetit B and Hieter P: The kinetochore and cancer: What's the connection? Curr Opin Cell Biol 17: 576-582, 2005.

30. Ghosh-Choudhury T, Loomans HA, Wan YW, Liu Z, Hawkins SM and Anderson ML: Abstract 3113: Hyperactivation of FOXM1 drives ovarian cancer growth and metastasis independent of the G2-M cell cycle checkpoint. Cancer Res 73 (Suppl): S3113, 2013.

31. Mercier PL, Bachvarova M, Plante M, Gregoire J, Renaud MC, Ghani K, Têtu B, Bairati I and Bachvarov D: Characterization of DOK1, a candidate tumor suppressor gene, in epithelial ovarian cancer. Mol Oncol 5: 438-453, 2011.

32. Lee YC, Huang CC, Lin DY, Chang WC and Lee KH: Overexpression of centromere protein K (CENPK) in ovarian cancer is correlated with poor patient survival and associated with predictive and prognostic relevance. PeerJ 3: e1386, 2015. 
33. Unoki $\mathrm{M}$, Brunet $\mathrm{J}$ and Mousli $\mathrm{M}$ : Drug discovery targeting epigenetic codes: The great potential of UHRF1, which links DNA methylation and histone modifications, as a drug target in cancers and toxoplasmosis. Biochem Pharmacol 78: 1279-1288, 2009.

34. Jenkins Y, Markovtsov V, Lang W, Sharma P, Pearsall D, Warner J, Franci C, Huang B, Huang J, Yam GC, et al: Critical role of the ubiquitin ligase activity of UHRF1, a nuclear RING finger protein, in tumor cell growth. Mol Biol Cell 16: 5621-5629, 2005.

35. Yan F, Wang X, Shao L, Ge M and Hu X: Analysis of UHRF1 expression in human ovarian cancer tissues and its regulation in cancer cell growth. Tumor Biol 36: 8887-8893, 2015.

36. Mantovani R: The molecular biology of the CCAAT-binding factor NF-Y. Gene 239: 15-27, 1999.

37. Gurtner A, Fuschi P, Magi F, Colussi C, Gaetano C, Dobbelstein M, Sacchi A and Piaggio G: NF-Y dependent epigenetic modifications discriminate between proliferating and postmitotic tissue. PLoS One 3: e2047, 2008

38. Yamanaka K, Mizuarai S, Eguchi T, Itadani H, Hirai H and Kotani H: Expression levels of NF-Y target genes changed by CDKN1B correlate with clinical prognosis in multiple cancers. Genomics 94: 219-227, 2009.

39. Garipov A, Li H, Bitler BG, Thapa RJ, Balachandran S and Zhang R: NF-YA underlies EZH2 upregulation and is essential for proliferation of human epithelial ovarian cancer cells. Mol Cancer Res 11: 360-369, 2013.

40. Zhang Y, Zhao FJ, Chen LL, Wang LQ, Nephew KP, Wu YL and Zhang S: MiR-373 targeting of the Rab22a oncogene suppresses tumor invasion and metastasis in ovarian cancer. Oncotarget 5: 12291-12303, 2014.

41. Shen J, Ambrosone CB, DiCioccio RA, Odunsi K, Lele SB and Zhao $\mathrm{H}$ : A functional polymorphism in the miR-146a gene and age of familial breast/ovarian cancer diagnosis. Carcinogenesis 29: 1963-1966, 2008.

42. Mullany LK, Liu Z, King ER, Wong KK and Richards JS: Wild-type tumor repressor protein 53 (Trp53) promotes ovarian cancer cell survival. Endocrinology 153: 1638-1648, 2012.

43. Wong KK, Lu KH, Malpica A, Bodurka DC, Shvartsman HS, Schmandt RE, Thornton AD, Deavers MT, Silva EG and Gershenson DM: Significantly greater expression of ER, PR, and ECAD in advanced-stage low-grade ovarian serous carcinoma as revealed by immunohistochemical analysis. Int $\mathbf{J}$ Gynecol Pathol 26: 404-409, 2007.
44. Wong KK, Izaguirre DI, Kwan SY, King ER, Deavers MT, Sood AK, Mok SC and Gershenson DM: Poor survival with wild-type TP53 ovarian cancer? Gynecol Oncol 130: 565-569, 2013.

45. Storms RW, Trujillo AP, Springer JB, Shah L, Colvin OM, Ludeman SM and Smith C: Isolation of primitive human hematopoietic progenitors on the basis of aldehyde dehydrogenase activity. Proc Natl Acad Sci USA 96: 9118-9123, 1999.

46. Sun P, Sehouli J, Denkert C, Mustea A, Könsgen D, Koch I, Wei L and Lichtenegger W: Expression of estrogen receptor-related receptors, a subfamily of orphan nuclear receptors, as new tumor biomarkers in ovarian cancer cells. J Mol Med (Berl) 83: 457-467, 2005

47. Halon A, Materna V, Drag-Zalesinska M, Nowak-Markwitz E, Gansukh T, Donizy P, Spaczynski M, Zabel M, Dietel M, Lage H and Surowiak P: Estrogen receptor alpha expression in ovarian cancer predicts longer overall survival. Pathol Oncol Res 17: 511-518, 2011

48. Liu Q, Chen Y, Pan JJ and Murakami T: Expression of protocadherin-9 and protocadherin-17 in the nervous system of the embryonic zebrafish. Gene Expr Patterns 9: 490-496, 2009.

49. Brasch J, Harrison OJ, Honig B and Shapiro L: Thinking outside the cell: How cadherins drive adhesion. Trends Cell Biol 22: 299-310, 2012

50. Bendas $\mathrm{G}$ and Borsig L: Cancer cell adhesion and metastasis: Selectins, integrins, and the inhibitory potential of heparins. Int J Cell Biol 2012: 676731, 2012.

51. Asad M, Wong MK, Tan TZ, Choolani M, Low J, Mori S, Virshup D, Thiery JP and Huang RY: FZD7 drives in vitro aggressiveness in Stem-A subtype of ovarian cancer via regulation of non-canonical Wnt/PCP pathway. Cell Death Dis 5: e1346, 2014.

52. Taylor DD and Gercel-Taylor C: MicroRNA signatures of tumor-derived exosomes as diagnostic biomarkers of ovarian cancer. Gynecol Oncol 110: 13-21, 2008.

53. Li Y, Yao L, Liu F, Hong J, Chen L, Zhang B and Zhang W: Characterization of microRNA expression in serous ovarian carcinoma. Int J Mol Med 34: 491-498, 2014 